\title{
CHiCAGO: robust detection of DNA looping interactions in Capture Hi-C data
}

\author{
Jonathan Cairns ${ }^{1 \dagger}$, Paula Freire-Pritchett ${ }^{1 \dagger}$, Steven W. Wingett ${ }^{1,2}$, Csilla Várnai ${ }^{1}$, Andrew Dimond ${ }^{1}$, Vincent Plagnol ${ }^{3}$, \\ Daniel Zerbino ${ }^{4}$, Stefan Schoenfelder ${ }^{1}$, Biola-Maria Javierre ${ }^{1}$, Cameron Osborne ${ }^{5}$, Peter Fraser ${ }^{1}$ \\ and Mikhail Spivakov ${ }^{1 *}$
}

\begin{abstract}
Capture $\mathrm{Hi}-\mathrm{C}(\mathrm{CHi}-\mathrm{C})$ is a method for profiling chromosomal interactions involving targeted regions of interest, such as gene promoters, globally and at high resolution. Signal detection in $\mathrm{CHi}-\mathrm{C}$ data involves a number of statistical challenges that are not observed when using other $\mathrm{Hi}-\mathrm{C}-$ like techniques. We present a background model and algorithms for normalisation and multiple testing that are specifically adapted to $\mathrm{CHi}-\mathrm{C}$ experiments. We implement these procedures in CHiCAGO (http://regulatorygenomicsgroup.org/chicago), an open-source package for robust interaction detection in $\mathrm{CHi}-\mathrm{C}$. We validate $\mathrm{CHiCAGO}$ by showing that promoter-interacting regions detected with this method are enriched for regulatory features and disease-associated SNPS.
\end{abstract}

Keywords: Gene regulation, Nuclear organisation, Promoter-enhancer interactions, Capture Hi-C, Convolution background model, $P$ value weighting

\section{Background}

Chromosome conformation capture (3C) technology has revolutionised the analysis of nuclear organisation, leading to important insights into gene regulation [1]. While the original $3 \mathrm{C}$ protocol tested interactions between a single pair of candidate regions ("one vs one"), subsequent efforts focused on increasing the throughput of this technology (4C, "one vs all"; $5 \mathrm{C}$, "many vs many"), culminating in the development of $\mathrm{Hi}-\mathrm{C}$, a method that interrogated the whole nuclear interactome ("all vs all") $[1,2]$. The extremely large number of possible pairwise interactions in $\mathrm{Hi}-\mathrm{C}$ samples, however, imposes limitations on the realistically achievable sequencing depth at individual interactions, leading to reduced sensitivity. The recently developed Capture $\mathrm{Hi}-\mathrm{C}(\mathrm{CHi}-\mathrm{C})$ technology uses sequence capture to enrich $\mathrm{Hi}-\mathrm{C}$ material for multiple genomic regions of interest (hereafter referred to as "baits"), making it possible to profile the global interaction profiles of many thousands of regions globally ("many vs all") and at a high resolution (Fig. 1) [3-7].

\footnotetext{
* Correspondence: mikhail.spivakov@babraham.ac.uk

${ }^{\dagger}$ Equal contributors

${ }^{1}$ Nuclear Dynamics Programme, Babraham Institute, Cambridge, UK

Full list of author information is available at the end of the article
}

$\mathrm{CHi}-\mathrm{C}$ data possess statistical properties that set them apart from other $3 \mathrm{C} / 4 \mathrm{C} / \mathrm{Hi}$-C-like methods. First, in contrast to traditional $\mathrm{Hi}-\mathrm{C}$ or $5 \mathrm{C}$, baits in $\mathrm{CHi}-\mathrm{C}$ comprise a subset of restriction fragments, while any fragment in the genome can be detected on the "other end" of an interaction. This asymmetry of $\mathrm{CHi}-\mathrm{C}$ interaction matrices is not accounted for by the normalisation procedures developed for traditional $\mathrm{Hi}-\mathrm{C}$ and $5 \mathrm{C}$ [8-10]. Secondly, CHi-C baits, but not other ends, have a further source of bias associated with uneven capture efficiency. In addition, the need for detecting interactions globally and at a single-fragment resolution creates specific multiple testing challenges that are less pronounced with binned $\mathrm{Hi}-\mathrm{C}$ data or the more focused $4 \mathrm{C}$ and $5 \mathrm{C}$ assays, which involve fewer interaction tests. Finally, $\mathrm{CHi}-\mathrm{C}$ designs such as Promoter $\mathrm{CHi}-\mathrm{C}$ and HiCap $[3-5,11]$ involve large numbers (many thousands) of spatially dispersed baits. This presents the opportunity to increase the robustness of signal detection by sharing information across baits. Such sharing is impossible in the analysis of $4 \mathrm{C}$ data that focuses on only a single bait and is of limited use in $4 \mathrm{C}$-seq containing a small number of baits [12-14].

These distinct features of $\mathrm{CHi}-\mathrm{C}$ data have prompted us to develop a bespoke statistical model and a 


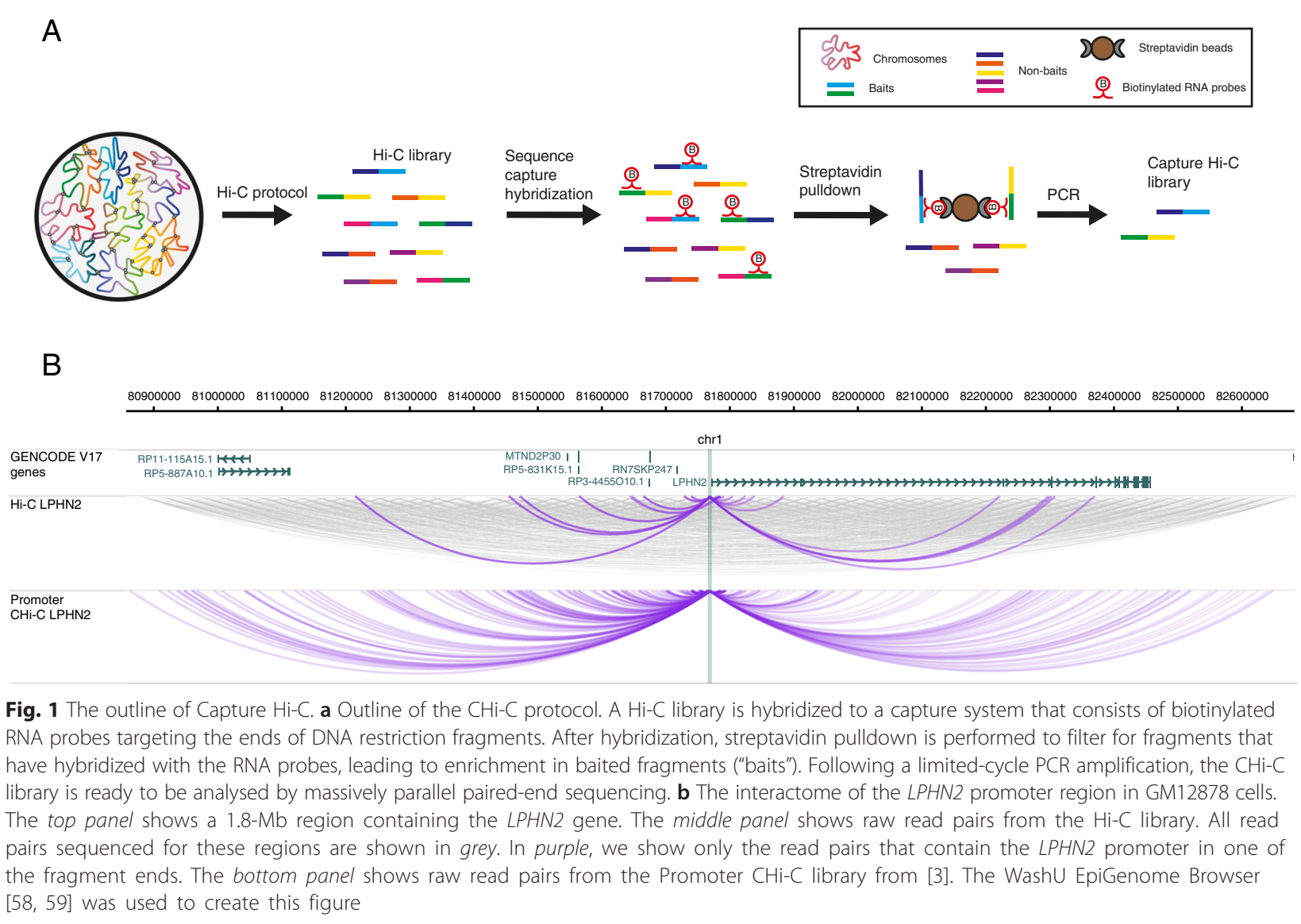

background correction procedure for detecting significant interactions in $\mathrm{CHi}-\mathrm{C}$ data at a single restriction fragment resolution. The algorithm, termed CHiCAGO ("Capture Hi-C Analysis of Genomic Organisation"), is presented here and implemented as an open-source $\mathrm{R}$ package. CHiCAGO features a novel background correction procedure and a two-component convolution background model accounting for both real, but expected, interactions as well as assay and sequencing artefacts. In addition, CHiCAGO implements a weighted false discovery control procedure that builds on the theoretical foundations of Genovese et al. [15]. This procedure specifically accommodates the fact that increasingly larger numbers of tests are performed at regions where progressively smaller numbers of interactions are expected.

We demonstrate the efficacy of CHiCAGO on two datasets: one from the human lymphoblastoid cell line GM12878 [3] (see Fig. 2 for examples) and another from mouse embryonic stem cells (mESCs) [4]. We further show that $\mathrm{CHiCAGO}$-detected interactions are enriched for regulatory regions and relevant disease-associated single-nucleotide polymorphisms (SNPs).

\section{Results}

\section{Methodological foundations of CHiCAGO A convolution background model for $\mathrm{Hi}-\mathrm{C}$ data}

The background levels in $\mathrm{CHi}-\mathrm{C}$ decrease as the genomic distance between the bait and other end increases (Fig. 3), as in other $3 \mathrm{C} / \mathrm{Hi}-\mathrm{C}$-like methods [6-10, 12, 13, 16, 17]. It is generally accepted that this effect reflects the reduction in the frequency of random collisions between genomic fragments owing to constrained Brownian motion of chromatin, in a manner consistent with molecular dynamics simulations [18]. We model the read counts arising from these "Brownian collisions" as a negative binomial random variable whose expected levels are a function of genomic distance, with further adjustment for bias resulting from the properties of individual fragments.

In addition to Brownian collisions, background in $\mathrm{CHi}-\mathrm{C}$ is generated by assay artefacts, such as sequencing errors. We model this "technical noise" component as a Poisson random variable whose mean depends on the properties of interacting fragments but is independent of genomic distance between them.

We further assume that these two sources of background counts are independent. Therefore, the combined 


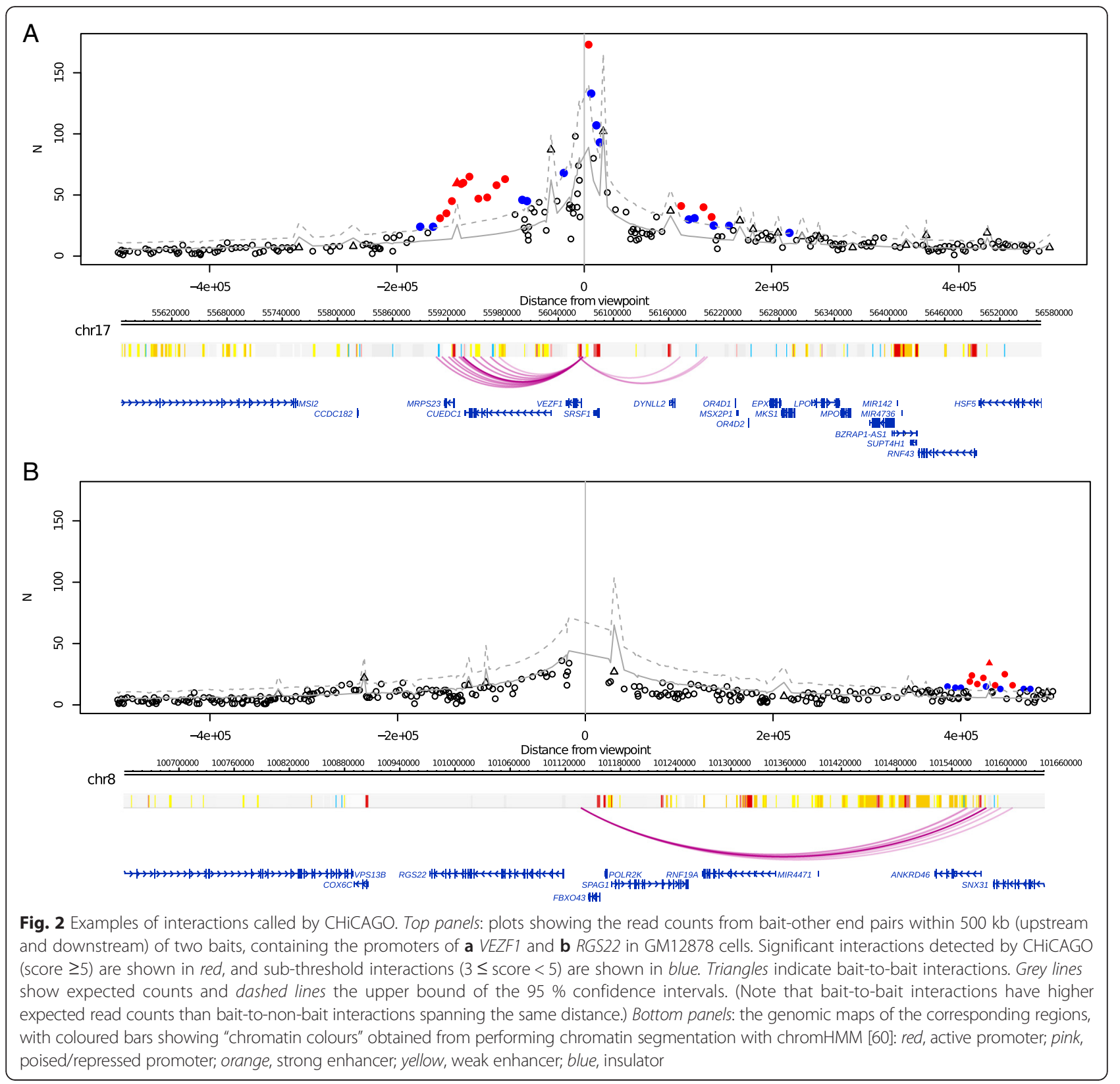

background distribution can be obtained as a convolution of negative binomial (Brownian collisions) and Poisson (technical noise) distributions that is known as the Delaporte distribution.

We first construct this null distribution from the data in a robust way, based on all possible fragment pairs (including those that have zero observed read counts). We then find the pairs with counts that greatly exceed the expected background level (Fig. 2; as described in the next section). The full mathematical specification of the algorithm is given in Additional file 1.

\section{Background estimation in asymmetrical interaction matrices}

A practical advantage of the two-component background model is that the Brownian and technical normalisation factors can be estimated on separate subsets of data, each of which predominantly represents only one background component.

The dependence of background levels on the distance between fragments is particularly apparent at relatively short genomic distances (up to $\sim 1-2 \mathrm{Mb}$ ), where the read counts considerably exceed those observed at longer ranges and for trans-chromosomal interactions. Thus, 


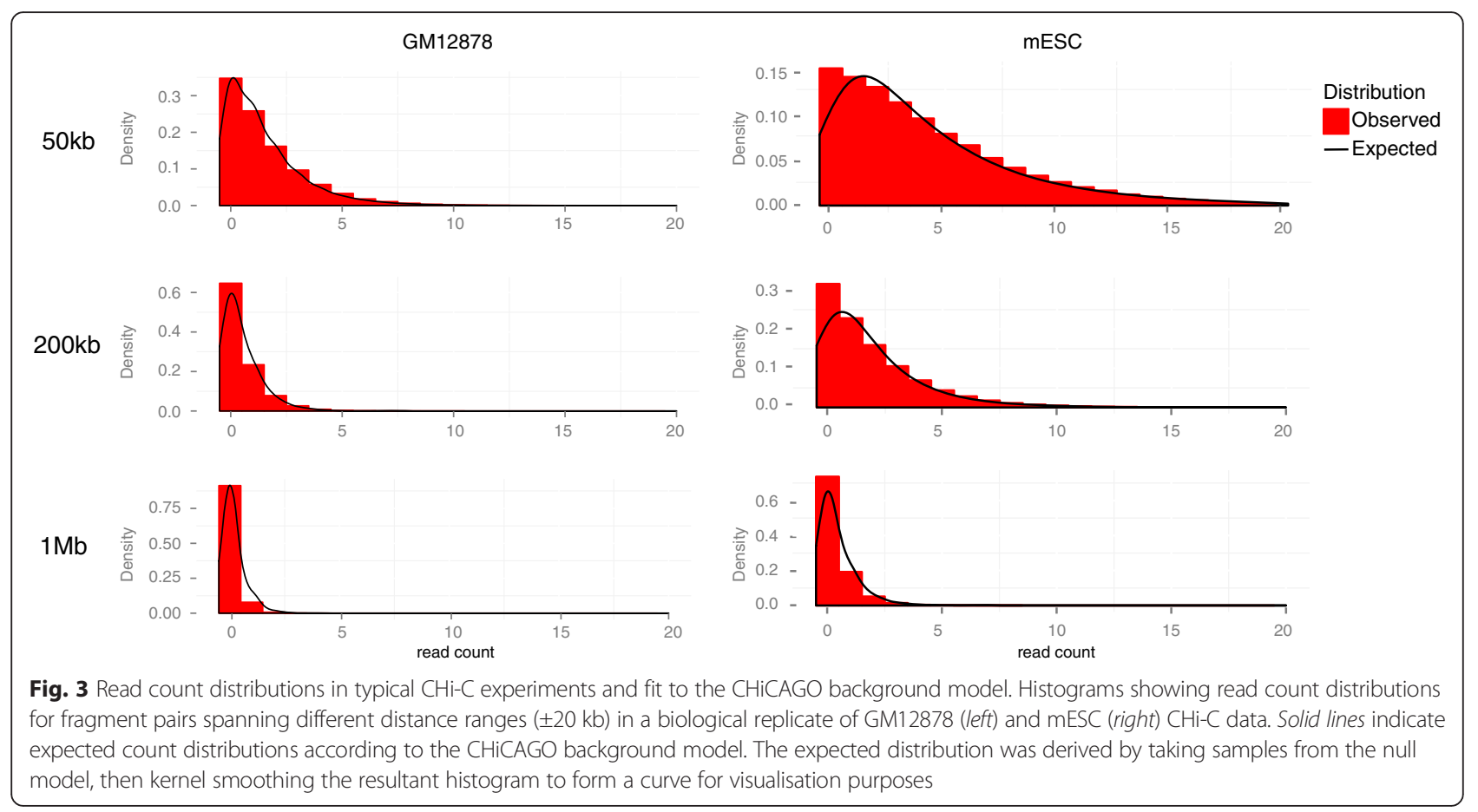

within this range, counts arising from Brownian collisions largely dominate over technical noise and, hence, the Brownian component can be estimated while ignoring the technical noise. By borrowing information across all interactions in this distance range, we can infer Brownian component parameters precisely (Fig. 4; Additional file 2: Figure S1). We follow Imakaev et al. [8] in assuming that fragment-level biases have a multiplicative effect on the expected read counts for each fragment pair. However, we estimate "bait-specific" and "other end-specific" bias factors differently, accounting for the asymmetry of $\mathrm{CHi}-\mathrm{C}$ interaction matrices.

The bait-specific factors reflect the technical biases of both $\mathrm{Hi}-\mathrm{C}$ and sequence capture, as well as local effects such as chromatin accessibility. We estimate these factors in a way that is robust to the presence of a small fraction of interactions in the data. Figure 4a provides examples of three baits with very diverse bias factors, illustrating that local read enrichment correlates with the bias factor.

Estimating other end-specific bias factors poses a challenge, as the majority of interactions are removed at the capture stage that enriches for only a small subset of interactions with baits. We assume that the overall fragmentlevel read count corresponding to trans-chromosomal pairs primarily reflects the general "noisiness" of a fragment (a similar approach has been taken independently in Dryden et al. [6]). While we do not preclude the presence of individual trans-chromosomal interaction signals, our reasoning that the overall per-fragment levels of transchromosomal pairs are dominated by noise is supported by evidence from $\mathrm{Hi}-\mathrm{C}$ and random ligation control data
(Additional file 2: Figure S2). We therefore pool fragments according to this property and estimate bias factors for each pool. As expected, bias factors are higher for fragments associated with higher numbers of transchromosomal read pairs (Fig. 4c). Similarly, baits detected at the "other ends" of bait-to-bait pairs had higher background levels than non-baits, as expected given the preferential recovery of "double-baited" ligation products at the capture stage.

In parallel, we compute the dependence between the Brownian background component and linear chromosomal distance (plotted in Fig. 4b for GM12878 CHi-C data). It can be seen that this dependence approximately follows a piecewise power law, consistent with previous studies on the subject, both theoretical and experimental $[18,19]$. We further show by cross-validation that the estimate of this dependence is stable (Additional file 2: Figure S3) and, therefore, unlikely to be influenced by bait-specific or interaction-specific signals.

To estimate the magnitude of technical noise, we again use the per-fragment total trans-chromosomal read pairs (see "Methods"). In doing so, we assume that the contribution of true signals from specific trans-chromosomal looping interactions, as well as from Brownian collisions between chromosomes to the total trans-chromosomal counts, is negligible for the reasons outlined above (Additional file 2: Figure S2). Indeed, as we see in Fig. 4d, the expected level of technical noise is typically a small fraction of a count.

The estimated parameters of both background components are then combined into the Delaporte distribution. 


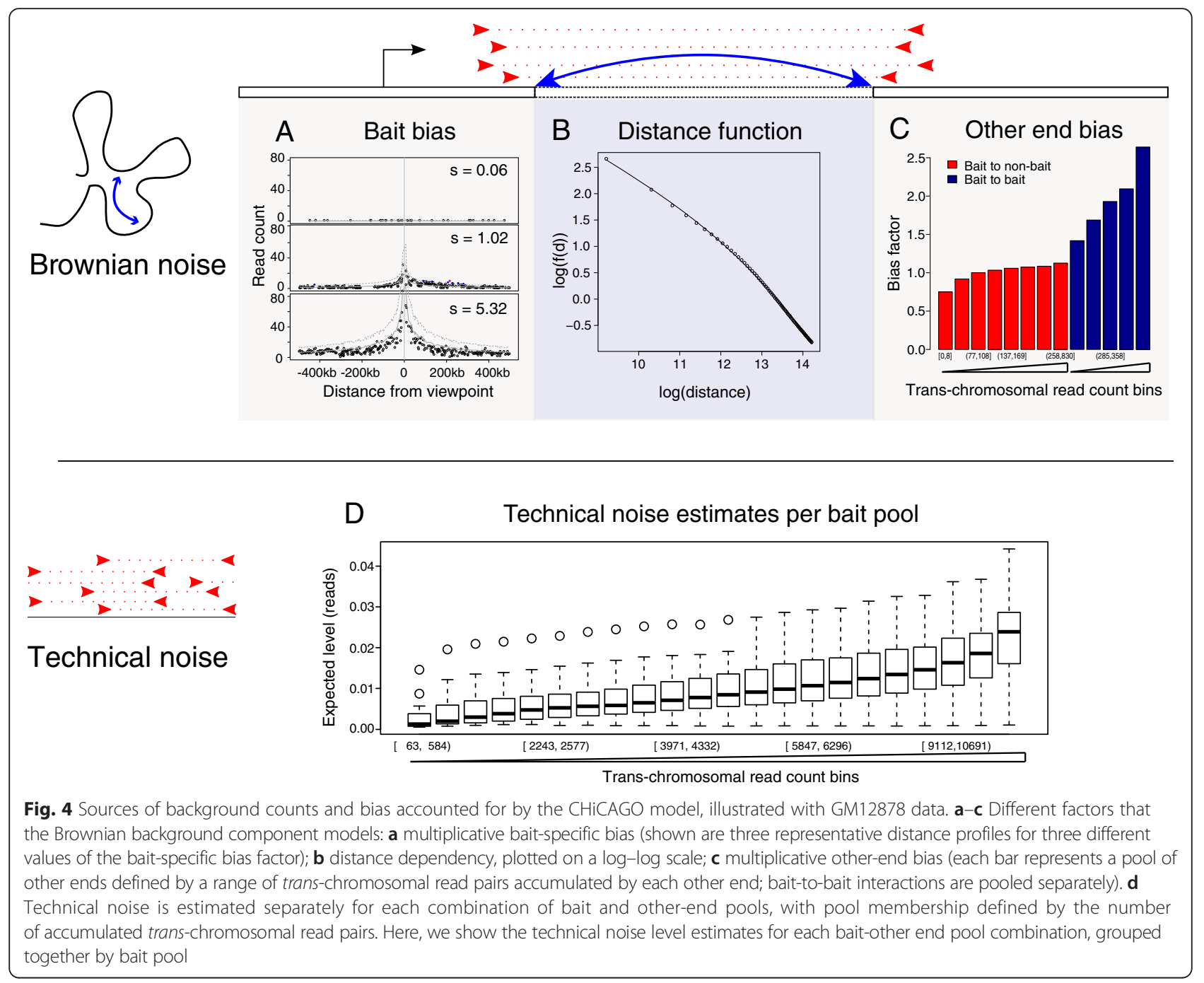

In Additional file 2: Figure S4 we show evidence that CHiCAGO's parameter estimation procedures are robust in the presence of undersampling; the implications of undersampling in $\mathrm{CHi}-\mathrm{C}$ data are further examined in the "Discussion". After appropriate normalisation and bias correction, we detect fragment pairs showing read coverage higher than expected under the Delaporte assumptions with a one-tailed hypothesis test.

\section{Weighted multiple testing correction for Capture Hi-C}

For a typical mammalian genome, we test billions of hypotheses-one for each possible bait-other end pair. As a result, the $p$ values must be corrected to account for multiple testing. Standard multiple testing procedures assume that interactions are equally likely at all distances. In $\mathrm{CHi}-\mathrm{C}$ data, however, we perform far more tests to verify the significance of interactions at large distances, where we would expect considerably fewer true interaction events. Consistent with this, the use of a single $p$ value threshold leads to results that consist mostly of erroneous distal and trans-chromosomal counts (Fig. 5b, c).

To address this issue, the long-range and transchromosomal interaction tests need to be more stringent than the short-range ones. We achieve this with an approach based on $p$ value weighting $[15,20]$. This procedure permits a smooth change of behaviour with distance, thereby bypassing the need to choose a hard distance threshold. Briefly, we assign each fragment pair a weight, estimating how probable it is that the fragments interact. The weights are then used to adjust the $p$ values (see Additional file 1 for full specification). $P$ value weighting can be seen as a simplified version of the empirical Bayesian treatment, with weights related to prior probabilities. One practical advantage of this method for our framework is that it avoids the need to make specific assumptions about the read count distribution of true interactions, which would be required for computing Bayes factors.

The optimal choice of weights depends on the relative abundance of true positives at each bait-other end 
A

Empirical probability of reproducible interaction

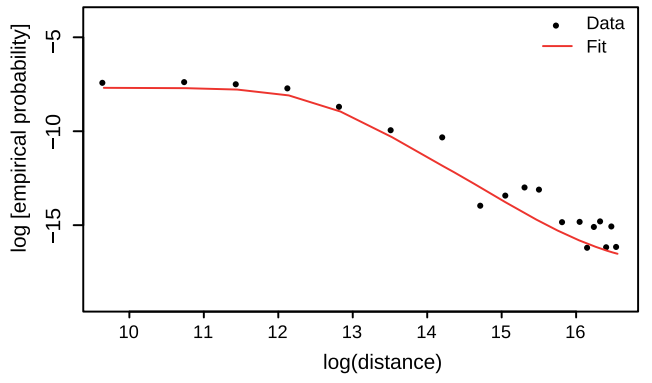

B

Interaction distance

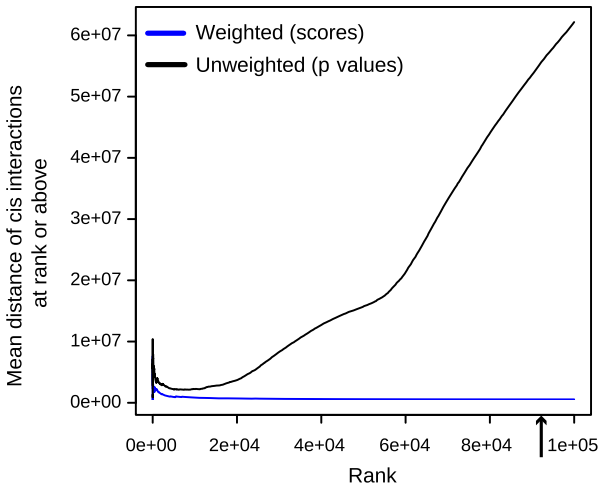

C

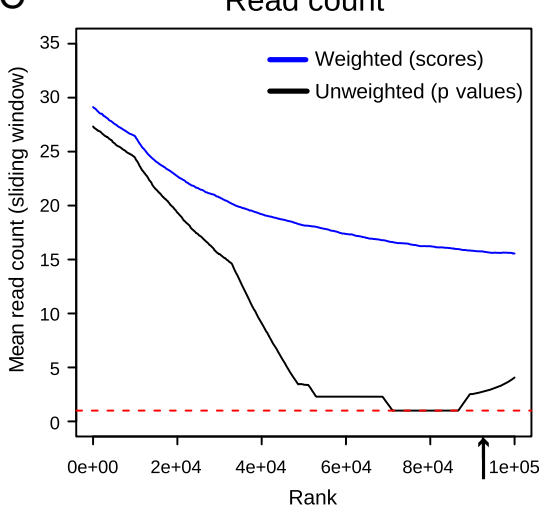

D

$\%$ Trans interactions

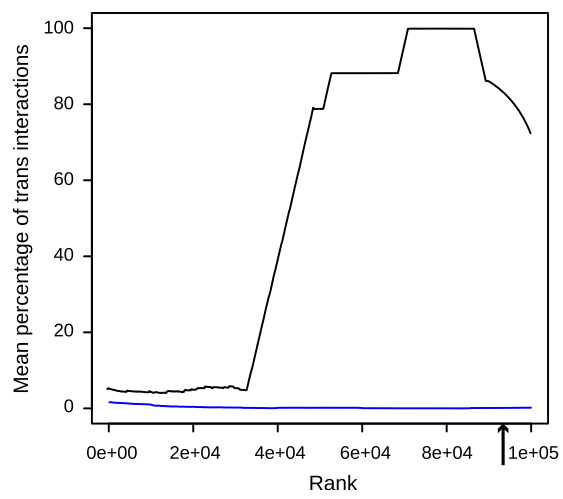

Fig. 5 CHiCAGO multiple testing approach schematic. a Empirical probability of reproducible interaction (used to generate weight profiles) as a function of interaction distance, generated on two replicates of GM12878 cells, assessed for the 100,000 top-scoring interactions. b-d The effects of applying $p$ value weighting to the GM12878 data. The arrow on the $x$-axis indicates the number of significant interactions called in the weighted data. Upon applying weighting, we see a decrease in the interaction distance amongst cis-interactions (b). $P$ value weighting increases the mean read count of called interactions (c) and decreases the prevalence of trans-chromosomal interactions (d)

distance. We estimate this abundance by assessing reproducibility across samples and fitting a bounded logistic curve to the observed reproducibility levels at different distances. Generally similar weight profiles were obtained in GM12878 cells and mESCs, and swapping them between these two datasets yielded highly correlated score profiles (Fig. 5a; Additional file 2: Figure S5). This is consistent with our expectation that weights are largely independent of specific cell type and organism given comparable genome sizes, as they predominantly reflect the overall distance distribution of true interactions. Emerging multi-replicate $\mathrm{CHi}-\mathrm{C}$ datasets will further refine our weight estimates and enable a more comprehensive assessment of their dependence on the particulars of the model system.

We illustrate the impact of the weighting procedure on GM12878 and mESC CHi-C data by comparing the 
properties of the 100,000 top-scoring interactions, called either with or without weighting. The reproducibility of interaction calls decreases with bait-other end distance (Fig. 5a; Additional file 2: Figure S5a). As a result, the "weighted" significant interactions generally span a much shorter range than the unweighted ones (Fig. 5b; Additional file 2: Figure S5b). This is consistent with the biological expectation that promoter-interacting regions, such as enhancers, are enriched in the relative vicinity of their targets. Another consequence of the weighting procedure is that the average read count is much higher in the weighted calls (Fig. 5c; Additional file 2: Figure S5c). Strikingly, many of the unweighted calls are based on only one read pair per interaction. As the vast majority of fragment pairs attract no reads at all, low $p$ values for single read-pair interactions are expected. However, due to the very large number of possible fragment pairs (approximately 18.5 billion in both the GM12878 and the mESC data), we still expect thousands of single read-count calls to be generated by technical noise. These spurious calls, the majority of which correspond to trans-chromosomal pairs (Fig. 5d; Additional file 2: Figure S5d), are generally nonreproducible and are therefore excluded by the weighting procedure.

In conclusion, the $p$ value weighting procedure implemented in CHiCAGO provides a multiple testing treatment that accounts for the differences in true positive rates at different bait-other end distances, thus improving the reproducibility of interaction calls.

\section{Promoter interactions detected by CHiCAGO: validation and key properties}

We validated CHiCAGO by assessing the functional properties of significant interactions detected with it in human GM12878 cells [3] and mESCs [4] under default settings and a score threshold of 5 . Table 1 displays summary statistics for each sample, showing the generally similar numbers of detected significant interactions, both overall and per bait, despite the differences in the organism and cell type between them.

\section{Enrichment for regulatory features}

We first assessed the enrichment of promoterinteracting fragments for histone marks associated with active (H3K4me1, H3K4me3, H3K27ac) and repressed (H3K27me3, H3K9me3) chromatin, as well as for the binding sites of CTCF, a protein with a well-established role in shaping nuclear architecture [21]. To this end, we compared the observed and expected numbers of promoter-interacting fragments overlapping with these features. To estimate the expected degree of overlap, we drew multiple permutations of the promoter-other end pairs not detected as interacting, such that the overall distribution of their spanned distances matched the distribution for the true interactions.

Figure 6 shows the observed and expected numbers of CHiCAGO other ends (yellow and blue bars, respectively) that overlap with the regulatory features in GM12878 and mESCs (panels a and b, respectively). Consistent enrichments over expected values were found for active histone marks (H3K4me1, H3K4me3, H3K27ac) in both cell types, in line with the expectation that looping interactions preferentially link promoters and remote regulatory regions such as enhancers. We also found that promoter-interacting fragments were strongly enriched for CTCF binding sites, as previously reported $[9,21]$. Interestingly, promoter-interacting fragments were also enriched for repressed chromatin marks, in particular for H3K27me3 in mESCs, supporting the role of Polycomb in shaping nuclear architecture in this cell type [5].

Assessing the enrichment of promoter-interacting fragments for known regulatory features can serve as a useful quality control for $\mathrm{CHi}-\mathrm{C}$ samples. To this end, CHiCAGO automatically generates enrichment bar plots similar to Fig. 6 for each sample, integrating interaction calls with user-specified genomic annotations, such as ChIP-seq peaks.

\section{Enrichment for genome-wide association study SNPs}

The majority of disease-associated SNPs identified in genome-wide association studies (GWAS) localise to non-coding regulatory regions, away from annotated

Table 1 The properties of CHiCAGO-detected interactions in human lymphoblastoid cell line GM12878 and mESCs

\begin{tabular}{lll}
\hline & GM12878 & mESC \\
\hline Number of captured baits & 22,076 & 22,459 \\
Total number of unique captured read pairs & Rep 1: 46,542,745 & Rep 1: 59,963,697 \\
& Rep 2: 82,026,534 \\
Number of significant interactions & Rep 3: $118,813,881,698$ & 94,148 \\
Mean number of significant interactions per bait & 88,667 & 4.19 \\
Median distance of cis-chromosomal interactions & 4.02 & 138,077 bp \\
\hline
\end{tabular}

Input read pairs are given per biological replicate (Rep). Default settings and a score threshold of 5 were used in interaction calling, performed jointly on all replicates 

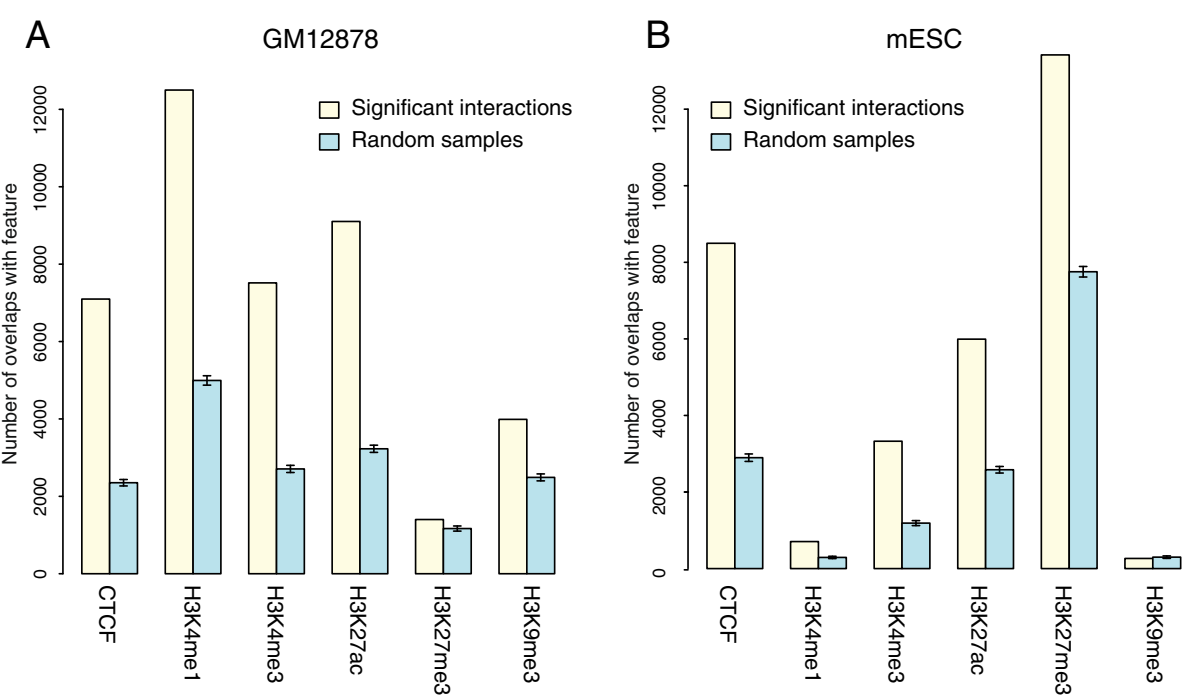

Fig. 6 Chromatin features of promoter-interacting fragments detected using CHiCAGO. Yellow bars indicate overlaps with cis-interacting fragments within $1 \mathrm{Mb}$ of promoter baits; blue bars indicate expected overlap values based on 100 random subsets of Hindlll fragments. These subsets were selected to have a similar distribution of distances from gene promoters as the interacting fragments. a GM12878 CHi-C data. Chromatin features are obtained from the ENCODE project [61]. b mESC CHi-C data. Chromatin features are obtained from the mouse ENCODE project [62]. These plots are generated automatically by the CHiCAGO pipeline. Error bars represent $95 \%$ confidence intervals

promoters, posing a significant challenge in identifying their putative target genes [22]. We asked whether promoter-interacting regions detected by CHiCAGO in human cells are enriched for GWAS SNPs, which would potentially reflect their presence in long-range regulatory sequences and thus suggest a putative functional role in disease.

We assessed the enrichment of promoter-interacting regions in GM12878 cells for sets of GWAS catalogue SNPs from Maurano et al. [22]. These sets reflect the grouping of GWAS traits into broader categories, such as autoimmune disease (AI), neurological/behavioural traits (NB) and kidney/liver/lung disorders (KLL). We used the software package GoShifter (Genomic Annotation Shifter) [23], which infers the significance of overlap by locally shifting genomic annotations (in our case, the "other ends" of CHiCAGO-detected promoter interactions), thus reducing the effect of genomic biases and linkage disequilibrium structure. We observed a significant enrichment of CHiCAGO "other ends" for SNPs associated with autoimmune diseases (GOShifter $p=0.001$ ) but not with kidney/liver/lung disorders $(p=0.876)$ or neurological/behavioural traits $(p=0.742)$. This selective enrichment for autoimmune SNPs is consistent with GM12878 being a lymphocyte-derived cell line and replicates the original findings of Mifsud et al. [3].

We further confirmed that the enrichment for AI disease-associated SNPs was specific to promoter-interacting fragments. We used the same approach as in the previous section to generate 100 random samples of distancematched "negative" (non-significant) interactions and tested the other ends of these interactions for SNP enrichment. The enrichment for AI-associated SNPs was selectively observed in the "true" set but not in the "negative" set and neither set was enriched for the NB- and KLL-associated SNPs (Fig. 7).

Taken together, these results demonstrate the power of using $\mathrm{CHi}-\mathrm{C}$ data to link GWAS SNPs with their putative target genes in a cell type-specific and high-throughput manner. We expect this to be one of the key applications of $\mathrm{CHi}-\mathrm{C}$ in future clinical studies.

\section{Capability to drive transgene expression in vivo}

TRIP (Thousands of Reporters Integrated in Parallel) is a novel experimental technique to assess the influence of local chromatin context on gene expression. In TRIP analysis, a barcoded transgene reporter is randomly integrated into thousands of genomic locations in parallel and the transcriptional activity at each location is then monitored. Here we integrated the published TRIP analysis dataset in mESCs [24] with the CHiCAGO mESC calls [4], comparing the transcriptional activity at promoter-interacting regions with the activity elsewhere, over a range of genomic distances.

Consistent with the observation from the original TRIP study, we found that the distance from the nearest promoter was a strong determinant of transgene expression levels (Fig. 8). However, transgenes mapping to promoter-interacting fragments consistently showed higher expression levels across the whole range of genomic distances, as confirmed by linear regression (effect size $=0.825 ;$ Wald test $p<0.001)$. This result provides 


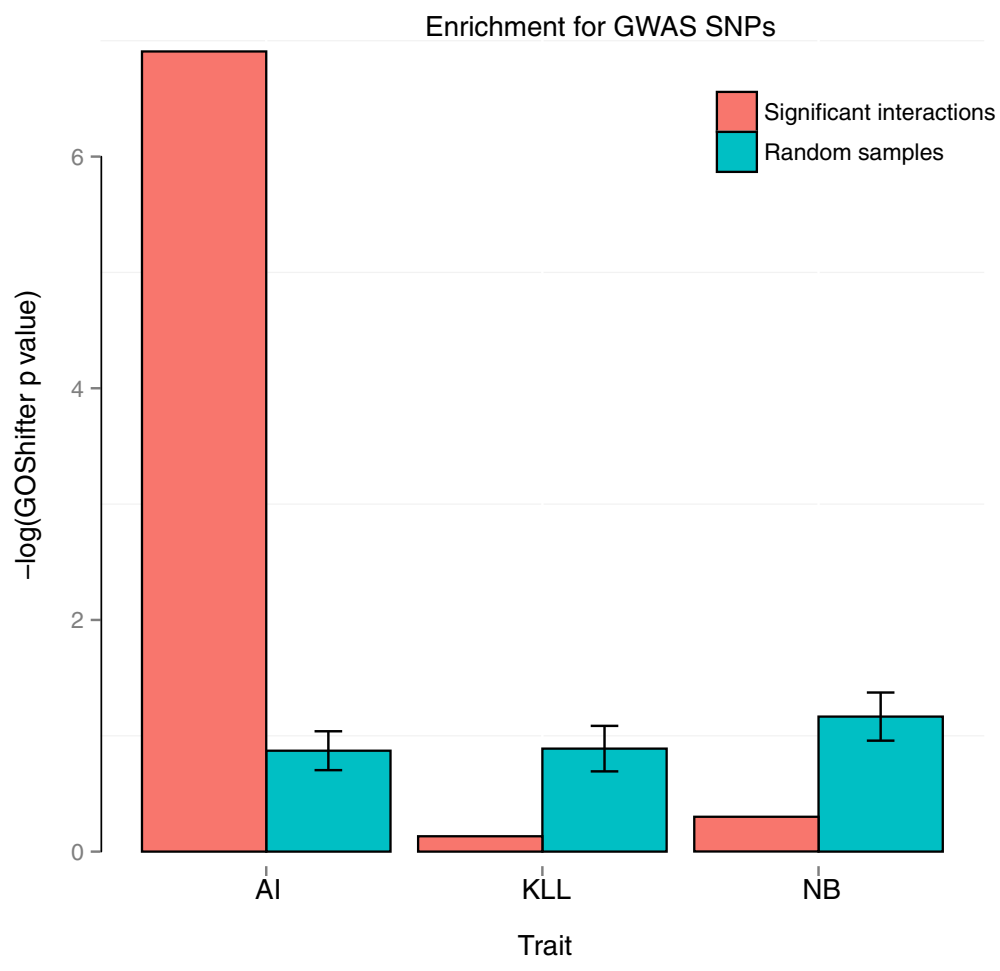

Fig. 7 Significant enrichment for GWAS SNPs at CHiCAGO-detected promoter-interacting regions in human lymphoblastoid cells. Enrichment for SNPs associated with autoimmune diseases (Al), and kidney/liver/lung (KLL) and neurological behaviour (NB) disorders [22] in the CHiCAGOdetected interactions in the GM12878 cell line. The bar plot shows $p$ values for the enrichment of each disorder; red bars indicate $p$ values computed in interacting fragments; blue bars indicate $p$ values computed in 100 random subsets of Hindlll fragments selected to have a similar distribution of distances from gene promoters as the interacting fragments. This analysis was performed using the software package GoShifter (Genomic Annotation Shifter) [23]

functional evidence that CHiCAGO-detected promoterinteracting fragments preferentially possess transcriptional regulatory activity.

\section{Promoter-promoter networks}

Interactions where both fragment ends are baited (referred to as "bait-to-bait interactions") represent contacts between gene promoters. These interactions are of special interest because they may help to identify sets of co-regulated genes recruited to either shared transcription factories [25] or repression networks such as those mediated by Polycomb proteins [5].

As an illustration of CHiCAGO's potential in identifying sets of co-regulated genes, we show CHiCAGOdetected bait-to-bait interactions involving histone promoters present on chromosome 6 in GM12878 cells (Fig. 9). We see that histone promoters frequently interact with other histone promoters, more so than with promoters of other genes in the same genomic region, consistent with previous observations $[4,26,27]$.

\section{Extremely long-range promoter interactions map within broader $\mathrm{Hi}$ - $\mathrm{C}$ contact regions}

We took advantage of the pre-capture $\mathrm{Hi}-\mathrm{C}$ dataset in mESCs [4] to compare CHiCAGO-detected interactions in Promoter $\mathrm{CHi}-\mathrm{C}$ with the broader-scale interaction signals detectable in $\mathrm{Hi}-\mathrm{C}$. The Promoter $\mathrm{CHi}-\mathrm{C}$ dataset has over tenfold higher coverage at promoters compared with the respective $\mathrm{Hi}-\mathrm{C}$ sample [4] and thus we would expect a corresponding increase in the sensitivity of detecting promoter-containing interactions. Consistent with this, while some stronger interactions in the short range $(<1 \mathrm{Mb})$ could be visually distinguished on $\mathrm{Hi}-\mathrm{C}$ interaction matrices (Fig. 10a), more than $80 \%$ of $\mathrm{CHi}-\mathrm{C}$ interactions in this range localised away from $\mathrm{Hi}-\mathrm{C}$ interacting regions detected with HOMER [28] at a 25$\mathrm{kb}$ resolution (Fig. 10b). In contrast, we found that more than $80 \%$ extremely long-range (>10 Mb) cis-chromosomal interactions and $45 \%$ trans-chromosomal interactions mapped within the broader (1 Mb-wide) $\mathrm{Hi}-\mathrm{C}$ contact areas (Fig. 10c). However, only a small minority of these megabase-scale contact areas contained $\mathrm{CHi}-\mathrm{C}$ interactions $(\sim 3 \%$ of $>10 \mathrm{Mb}$ cis-chromosomal and $\sim 0.5 \%$ 


\section{Enrichment for regions capable of driving transgene expression in vivo}

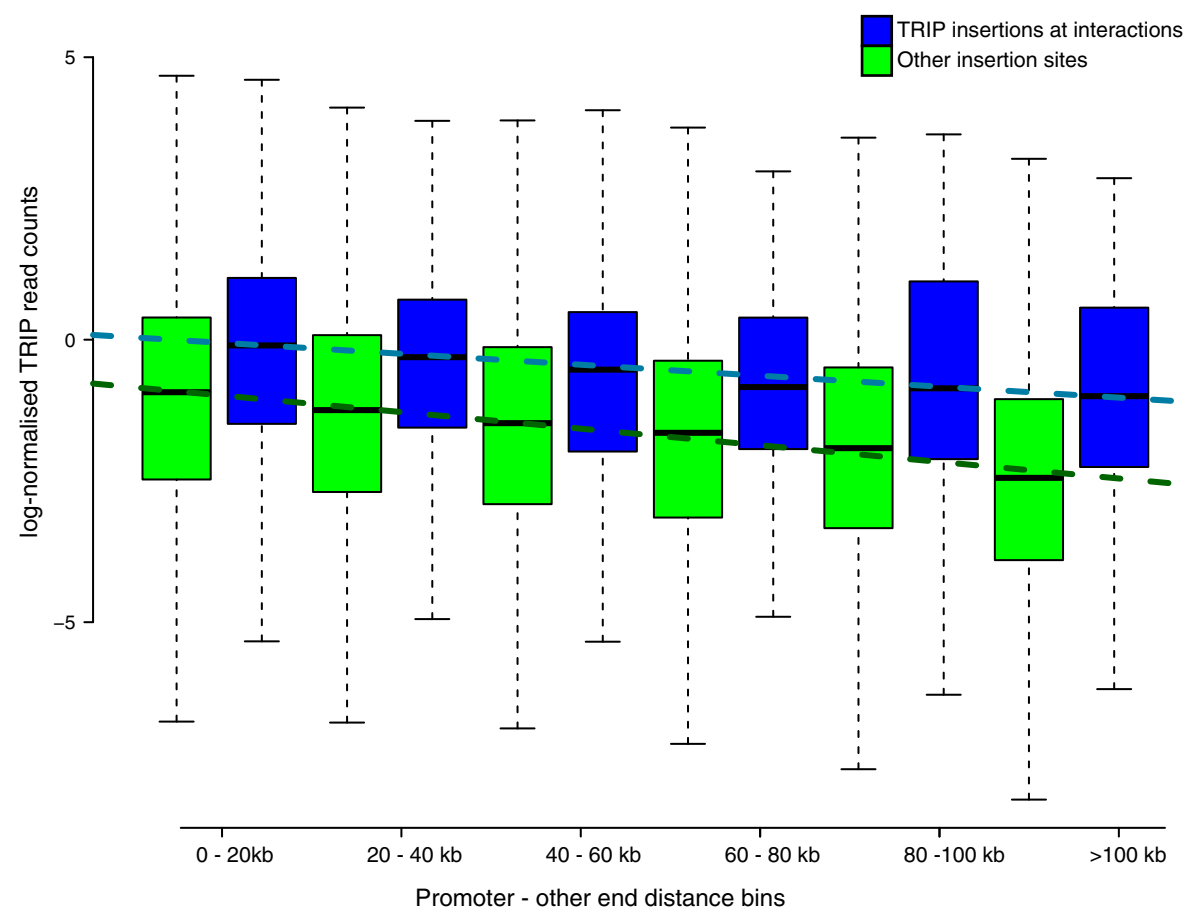

Fig. 8 Enrichment of promoter-interacting fragments for regions capable of driving transgene expression in mESCs. TRIP (Thousands of Reporters Integrated in Parallel) assesses the influence of local chromatin context on gene expression. This is achieved by integrating a barcoded transgene reporter into thousands of genomic locations in parallel and monitoring the transcriptional activity at each location [24]. Normalised RNA read counts from reporter insertions are separated according to (i) their overlap with Hindlll fragments engaging or not in interactions; (ii) their promoter-other end distance. For non-interacting Hindlll fragments, distance is measured from the nearest promoter in the linear sequence. Blue and green box plots indicate read count summary statistics for promoter-interacting and non-interacting Hindlll fragments, respectively. Each dashed line shows the regression of median log-normalised read counts against promoter-other end distance bin, considering promoter-interacting (blue) and non-interacting (green) Hindlll fragments separately

trans-chromosomal, as illustrated in Fig. $10 \mathrm{~d}$ and Additional file 2: Figure S6). Taken together, these results are consistent with a high specificity and resolution of $\mathrm{CHiCAGO}$ long-range interaction calling. At the same time, they warrant a further examination of the relationship between specific looping interactions and higher-order chromosomal contacts.

\section{Discussion}

In this paper, we present the CHiCAGO algorithm for Capture $\mathrm{Hi}-\mathrm{C}$ analysis and demonstrate its efficacy in detecting interactions enriched for regulatory chromatin features and relevant GWAS SNPs.

Our approach is based on the assumption that "significant" interactions emerge as outliers on a distancedependent local background profile. This assumption is shared by most other tools for interaction detection in $3 \mathrm{C}$-like data and seems reasonable for the purposes of identifying regulatory interactions. Indeed, it can be expected that regulatory events such as transcription factor binding will stabilise the chromatin loop, leading to interaction frequencies or retention times beyond those generated by random collisions due to Brownian motion. This expectation is supported by the observation that CHiCAGO-detected interactions are selectively enriched for regulatory chromatin features, even when located in regions with high background interaction levels.

While the conceptual interpretation of "significant" interactions is shared between CHiCAGO and algorithms developed for other types of $4 \mathrm{C}$ and $\mathrm{Hi}-\mathrm{C}$ data, there are key differences in terms of the underlying background model, the normalisation strategy and the multiple testing procedure.

Existing tools model Hi-C background with a broad range of distributions, both discrete (binomial [16, 29], negative binomial [6]) and continuous (Weibull [7, 9], normal [13]). In CHiCAGO, we instead opted for a twocomponent convolution model that incorporates two count distributions: a negative binomial and a Poisson. In doing so, we were motivated by the fact that distancedependent Brownian collisions and technical variability are two distinct background count-generating processes whose properties are best learned separately on different subsets of data. Indeed, signals from Brownian collisions 


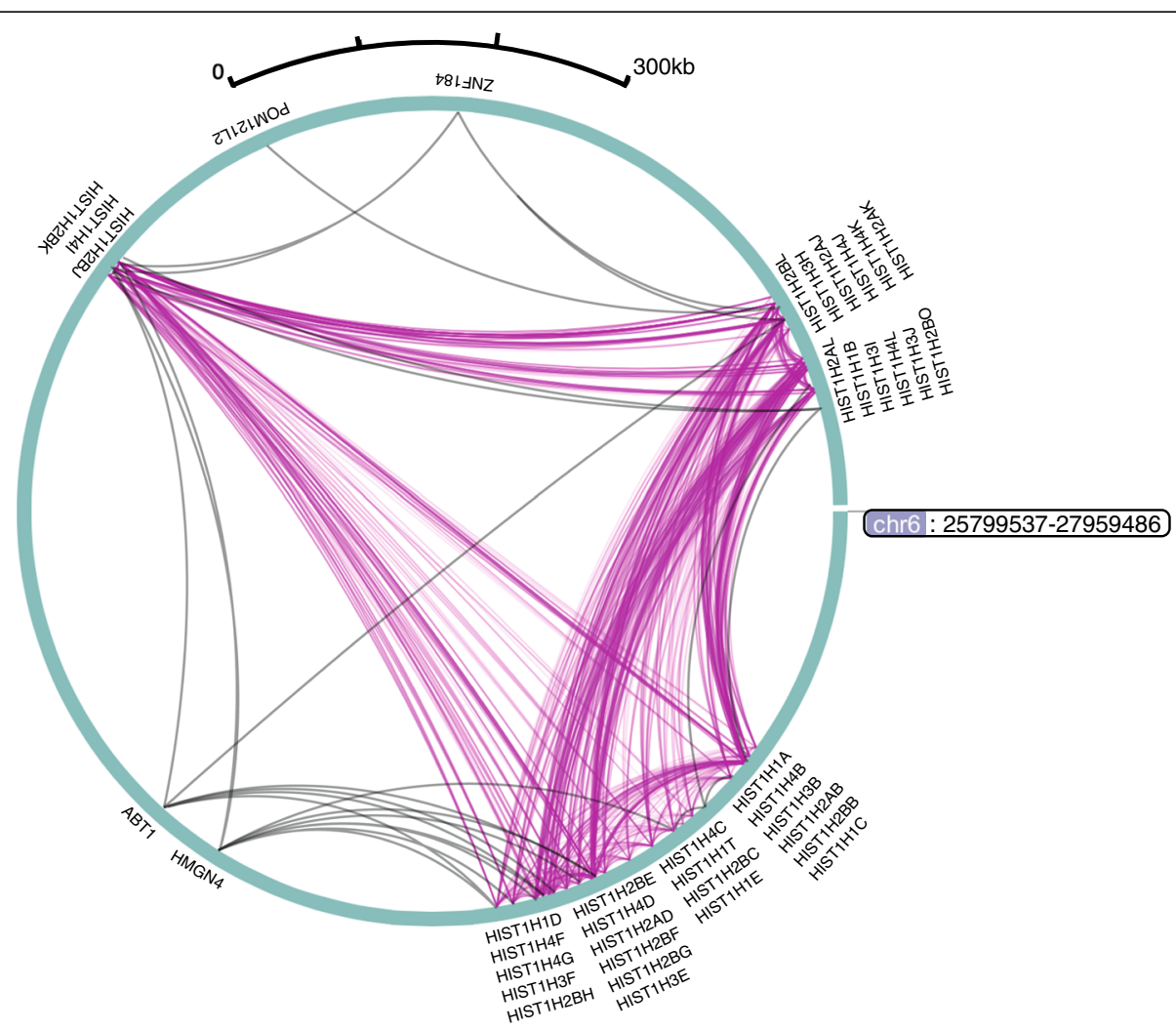

Fig. 9 Circlet view of promoter-promoter interactions for histone genes in GM12878 cells. Interactions where histone gene promoters are engaged at both fragment ends are shown in dark magenta. Interactions where histone gene promoters are interacting with non-histone gene promoters are shown in grey. The WashU EpiGenome Browser $[58,59]$ was used to create this figure

ostensibly dominate the background at short distances, to the extent that technical variability is barely detectable. In contrast, at large linear distances between fragments, Brownian collisions are too weak for their count distribution to be estimated directly. Thus, we infer this distribution by extrapolation.

Borrowing information across baits to learn the background model, as CHiCAGO does, requires careful normalisation across interactions. While $\mathrm{Hi}-\mathrm{C}$ background depends on a number of known parameters, such as fragment length and GC content [10], we, along with others $[7,8,30]$, have opted to avoid any specific assumptions about noise structure, particularly given the increased complexity and asymmetric nature of capture $\mathrm{Hi}-\mathrm{C}$ noise compared with conventional Hi-C. Assuming that interactions are subject to multiplicative bait- and other-end-specific bias, as we did in learning the Brownian background component, parallels the assumptions of the $\mathrm{Hi}-\mathrm{C}$ iterative correction approach by Imakaev et al. [8] and is generally consistent with data from molecular dynamics simulations of chromatin fibres [18]. In modelling technical noise, we assumed it to be reflected in the numbers of trans-chromosomal interactions involving the same fragment. A similar strategy has been applied independently in a recently published Capture $\mathrm{Hi}-\mathrm{C}$ study [6]; the same authors also proposed an iterative correction algorithm for Capture Hi-C data [7] (software not publicly released) that may complement the approaches taken here.

Multiple testing issues are important in genomic analyses and, in attempting to address these issues, a number of bespoke approaches have been developed [20,31]. The specific challenge of multiple testing in $\mathrm{Hi}-\mathrm{C}$ data is that we expect the fractions of true positives to vary depending on the genomic distance between the fragments; in fact, the majority of tests are performed with interactions spanning large distances or spanning different chromosomes, where true positive signals are least expected. CHiCAGO's multiple testing procedure is based on the $p$ value weighting approach by Genovese et al. [15], which is a generalisation of a segment-wise weighting procedure by Sun et al. [32]. These approaches have been used successfully to incorporate prior knowledge in GWAS [33-35] and are emerging in functional genomics analyses [36, 37]. In using the reproducibility of significant calls across replicates as an estimate of the relative true positive rate, we have taken inspiration from the irreproducible discovery rate (IDR) approach [38] 


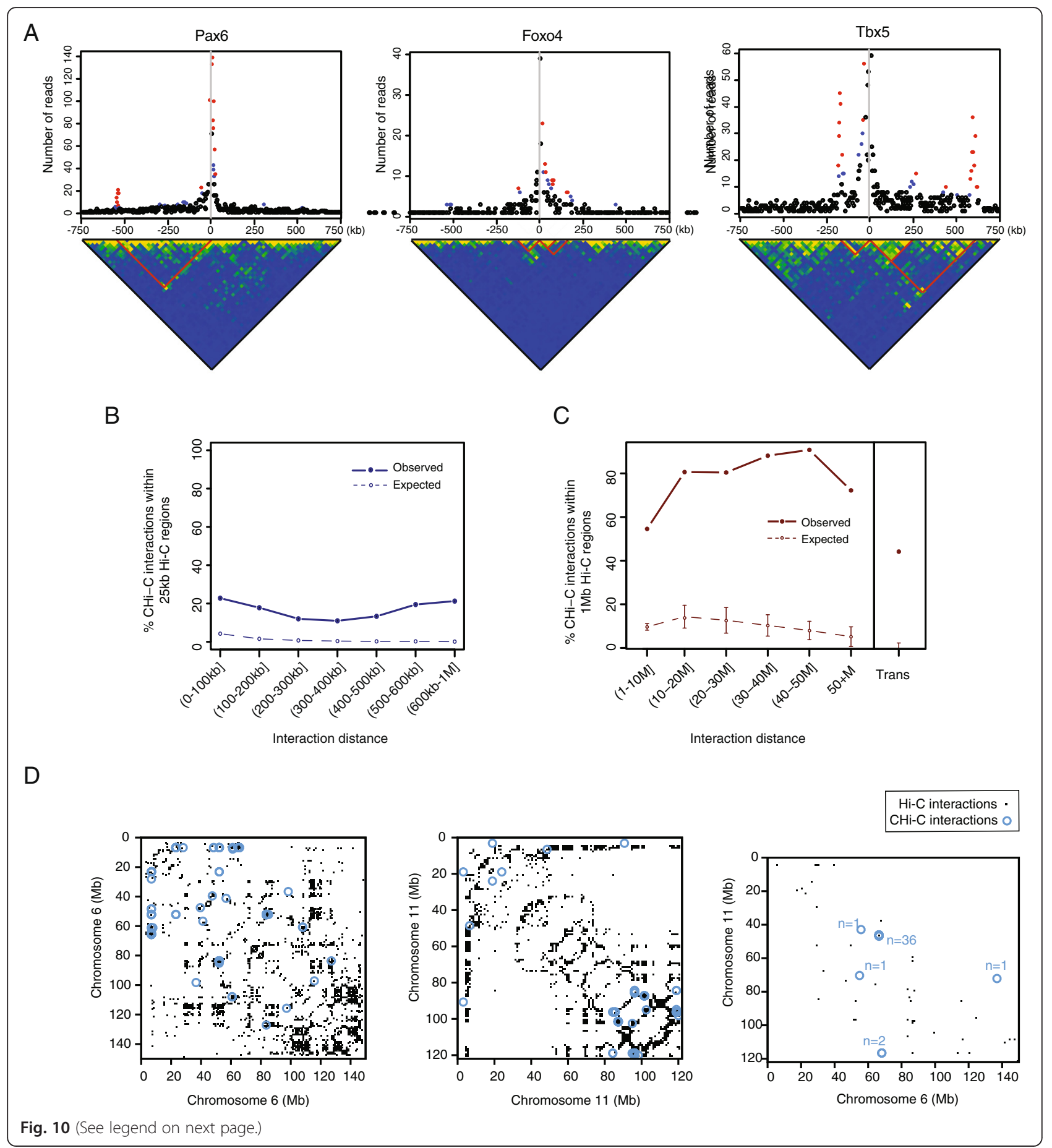


(See figure on previous page.)

Fig. 10 Comparison of interactions detected in $\mathrm{CHi}-\mathrm{C}$ and $\mathrm{Hi}-\mathrm{C}$ data. a Top panels: plots showing the read counts from bait-other end pairs within $750 \mathrm{~kb}$ (upstream and downstream) of three baits, containing the Pax6, Foxo4 and Tbx5 promoters (from left to right). Significant interactions detected by CHiCAGO (score $\geq 5$ ) are shown in red, and sub-threshold interactions $(3 \leq$ score $<5$ ) are shown in blue. Bottom panels: raw Hi-C matrices at $25-\mathrm{kb}$ bin resolution within the corresponding 1.5-Mb regions. The bottom corners of the red lines indicate example bin pairs, within which significant interactions were detected in the $\mathrm{CHi}-\mathrm{C}$ data. b Mapping of short-range $(<1 \mathrm{Mb}) \mathrm{CHi}-\mathrm{C}$ interactions within $25-\mathrm{kb}$ interacting bins detected in the $\mathrm{Hi}-\mathrm{C}$ data. Filled circles show the observed fraction of $\mathrm{CHi}-\mathrm{C}$ interactions mapping within the Hi-C interacting bins; open circles show the expected fraction estimated by a permutation strategy accounting for genomic structure (see "Methods" for details). The standard deviations across 100 permutations are not shown as they are smaller than point size. c Mapping of long-range (>1 Mb) $\mathrm{CHi}-\mathrm{C}$ interactions within 1-Mb interacting bins detected in the $\mathrm{Hi}-\mathrm{C}$ data. Filled circles show the observed fraction of long-range cis-and trans-chromosomal interactions detected in the $\mathrm{CHi}-\mathrm{C}$ data that map within the $\mathrm{Hi}-\mathrm{C}$ interacting bins. Open circles show the expected fraction estimated by a permutation strategy accounting for genomic structure (see "Methods" for details). Error bars show standard deviation across 100 permutations. d The overlap of long-range (>5 Mb) interacting fragment pairs detected in $\mathrm{CHi}-\mathrm{C}$ data (blue circles) and interacting 1-Mb bin pairs detected in the $\mathrm{Hi}-\mathrm{C}$ data (black squares) on chromosomes 6 (left) and 11 (centre) and for trans-interactions between these chromosomes (right). All panels present pre-capture mESC Hi-C data from [4]

used to determine peak signal thresholds in other types of genomics data, such as ChIP-seq.

Note that, in this setting, IDR cannot be used verbatim for choosing signal thresholds, as the relationship between Capture Hi-C signal and reproducibility does not satisfy IDR assumptions, likely because of undersampling issues (not shown). Importantly, conventional false discovery rate (FDR)-based approaches for multiple testing correction [39] are also unsuitable for these data. Indeed, $\mathrm{CHi}-\mathrm{C}$ observations (read-pair counts) are discrete and many of them are equal to either zero or one. This leads to a highly non-uniform distribution of $p$ values under the null, violating the basic assumption of conventional FDR approaches. The "soft-thresholding" approach used in CHiCAGO shifts the -log-weighted $p$ values such that non-zero scores correspond to observations, where the evidence for an interaction exceeds that for a pair of near-adjacent fragments with no reads. More robust thresholds can then be chosen based on custom criteria, such as maximising enrichment of promoter-interacting fragments for chromatin features (Fig. 6; a user-friendly function for this analysis is provided as part of the Chicago $\mathrm{R}$ package - see the package vignette provided as Additional file 3). Based on this approach, we chose a signal threshold of 5 for our own analyses.

The undersampled nature of $\mathrm{CHi}-\mathrm{C}$ data (particularly at longer distance ranges), although robustly handled by CHiCAGO, may lead to significant sensitivity issues when using thresholded interaction calls in comparative analyses. We therefore suggest performing comparisons based on the continuous score range. Potentially, differential analysis algorithms for sequencing data (such as DESeq2 [40]) may also be used to formally compare the enrichment at CHiCAGO-detected interactions between conditions at the count level, although power will generally be a limiting factor. As undersampling drives down the observed overlap of interactions called on different samples (Additional file 2: Figure S4c), methods such as $[41,42]$ may be considered for formally ascertaining the consistency between datasets. Additional filtering based on the mean number of reads per detected interaction (e.g., removing calls whose mean $\mathrm{N}$ is below 10 reads) will also reduce the impact of undersampling on the observed overlap, but at the cost of decreasing the power to detect longer-range interactions.

The $p$ value weighting approach used here is similar in spirit to an empirical Bayesian treatment, with the $p$ value weights related, but not identical, to prior probabilities. Bayesian approaches are widely used (including, recently, for signal detection in conventional $\mathrm{Hi}-\mathrm{C}$ [43]) and the Bayes factors and posterior probabilities they generate are potentially more intuitive than weighted $p$ values. However, the $p$ value weighting approach used here has the advantage of not making any specific assumptions about the read distributions of "true interactions", beyond their having a larger mean. Both approaches open the opportunity of incorporating prior knowledge, beyond the dependence of reproducibility on distance-for example, taking into account the boundaries of topologically associated domains (TADs) [44], higher-order contact domains and chromosomal territories. We choose not to do this currently because the exact relationship between these genomic properties and looping interactions still requires further investigation, and incorporating these relationships a priori prevents their investigation in post hoc analyses. Active research in this area makes it likely that much more will be known about the determinants of loop formation in the near future, enabling a more extensive use of prior knowledge in interaction detection, potentially with a formal Bayesian treatment.

The downstream analyses of CHiCAGO results provided in this paper confirm the enrichment of promoter-interacting regions for regulatory features and disease-associated variants. These results demonstrate the enormous potential of $\mathrm{CHi}-\mathrm{C}$ for both functional genomics and population genetics, and this assay will likely be applied in multitudes of other cell types in the near future. Therefore, user-friendly, open-source software for robust 
signal detection in these challenging data will be a welcome addition to the toolkits of many bioinformaticians and experimentalists alike. We have developed CHiCAGO with the view of addressing this need. Furthermore, we expect the statistical foundations of CHiCAGO, particularly the convolution background model and the multiple testing procedure, to be potentially useful in a broader range of Hi-C-related assays.

\section{Conclusions}

The publicly available, open-source CHiCAGO pipeline presented here [45] produces robust and interpretable interaction calls in $\mathrm{CHi}-\mathrm{C}$ data. Promoter-interacting fragments identified using this algorithm are enriched for active chromatin features, GWAS SNPs and regions capable of driving transgene expression, indicative of regulatory looping interactions. While developed specifically for $\mathrm{CHi}-\mathrm{C}$, the statistical principles of CHiCAGO are potentially applicable to other $\mathrm{Hi}$-C-based methods.

\section{Methods}

\section{Sample pre-processing}

The publicly available HiCUP pipeline $[46,47]$ was employed to process the raw sequencing reads. This pipeline was used to map the read pairs against the mouse (mm9) and human (hg19) genomes, to filter experimental artefacts (such as circularized reads and religations) and to remove duplicate reads. For the $\mathrm{CHi}-\mathrm{C}$ data, the resulting BAM files were processed into $\mathrm{CHi}$ CAGO input files, retaining only those read pairs that mapped, at least on one end, to a captured bait. The script bam2chicago.sh, used for this purpose, is available as part of the chicagoTools suite [45].

\section{The CHiCAGO algorithm}

A full description of the algorithm is given in Additional file 1. A tutorial on using the CHiCAGO package (the "vignette") is provided in Additional file 3.

Briefly, to combine replicates, a "reference" replicate is created by taking the geometric mean of each fragment pair's count across samples. Sample size factors are calculated by taking the mean ratio to the "reference" replicate, in a manner similar to the sample normalisation strategy implemented in DESeq [48]. Final counts are derived as the rounded weighted sum of counts across replicates, where the weights are the sample size factors.

Background from Brownian collisions is assumed to have negative binomial distribution, with mean $s_{i} s_{j} f\left(d_{i j}\right)$ and dispersion $r$, where $i$ indexes over other ends and $j$ indexes over baits.

Estimation of $s_{i}, s_{j}, f(d)$ and $r$ is performed in "proximal bins"-by default, 20-kb bins that span the first $1.5 \mathrm{Mb}$ around each bait.

The distance function $f(d)$ is estimated as follows:
- For each bait, take all of the other ends in a distance bin to get a mean count for that bin.

- $f(d)$ is estimated in a distance bin by taking the geometric mean of the bin counts at that distance, across all baits.

- To interpolate $f(d)$ from these point estimates, we use a maximum likelihood cubic fit on a log-log scale.

- Outside of this distance range, we extrapolate linearly, assuming continuity of $f$ and its first derivative.

The bait-specific scaling factors, $s_{j}$, are estimated by considering each mean bin count divided by $f(d)$, then taking the median of this ratio, across all bins associated with a bait. The other end-specific scaling factors, $s_{i}$, are estimated similarly but with the other ends pooled together (the pools are chosen such that their content ends have similar numbers of trans-chromosomal counts) so that there is enough information for a precise estimate. The dispersion, $r$, is estimated using standard maximum likelihood methods.

The technical noise is assumed to have Poisson distribution, with mean $\lambda_{i j}$. $\lambda_{i j}$ is estimated from trans-chromosomal counts-again, first pooling fragments by the number of trans-chromosomal counts they exhibit. Specifically, to estimate the technical noise level for a putative interaction between a bait in pool A and an other end in pool B, we count the number of interactions that span between pools $\mathrm{A}$ and $\mathrm{B}$ and divide this by $|\mathrm{A}||\mathrm{B}|$, the total number of bait-other end fragment pairs from those pools.

$P$ values are called with a Delaporte model, representing the sum of two variables: a negative binomial variable with mean $s_{i} s_{j} f\left(d_{i j}\right)$ and dispersion $r$, and a Poisson variable with mean $\lambda_{i j}$. A four-parameter bounded logistic regression model is assumed for $p$ value weighting (see the next section and Additional file 1 for more information).

The final CHiCAGO score is obtained from softthresholding the $-\log$ (weighted $p$ value). Specifically, the score is $\max \left(-\log (p)+\log (w)-\log \left(w_{\text {max }}\right), 0\right)$, where $w_{\text {max }}$ is the maximum attainable weight, corresponding to zero distance. For the downstream analyses in this paper, interactions with $\mathrm{CHiCAGO}$ scores $\geq 5$ were considered as "significant interactions".

\section{$P$ value weighting parameter estimation}

The $p$ value weighting function has four parameters: $\alpha$, $\beta, \gamma$ and $\delta$ (full details are given in Additional file 1). We can estimate these parameters from a candidate data set provided that it has multiple biological replicates, as follows. We split the data into subsets that contain approximately equal numbers of baits (by default, five 
subsets are used.) The reproducible interactions are defined as those where the stringent threshold of $\log (p)<-10$ is passed in all biological replicates. Now, for each subset, we take a series of genomic distance bins (with the default breaks occurring at $0,31.25 \mathrm{~kb}$, $62.5 \mathrm{~kb}, 125 \mathrm{~kb}, 250 \mathrm{~kb}, 500 \mathrm{~kb}, 1 \mathrm{Mb}, 2 \mathrm{Mb}, 3 \mathrm{Mb}$, $4 \mathrm{Mb}, \ldots, 16 \mathrm{Mb}$ ), and we calculate the proportion of reproducible interactions out of the total number of possible interactions. The maximum likelihood estimates are calculated for each model parameter using standard optimization methods [49]. Final parameter estimates are obtained by taking the median across the estimates from each subset. The two replicates of mESC data [4] were used for estimating weights. For GM12878 [3], the first replicate was not used for weight estimation as it led to unstable estimation. This was likely due to the poorer quality of this replicate compared with the other two, consistent with its higher cis/trans-chromosomal count ratios (data not shown). Recommendations on diagnosing unstable estimates are provided in the $\mathrm{R}$ package vignette (Additional file 3).

\section{The Chicago R package}

CHiCAGO was implemented as a package for the statistical environment $\mathrm{R}$ [50] taking advantage of the data.table objects [51] to optimise for both speed and memory. The fully documented R package "Chicago" and the tutorial data package "PCHiCdata" are publicly available [45] under Artistic Licence 2.0 and are part of Bioconductor release 3.3+ [52, 53]. A documented set of supplementary scripts (chicagoTools) for data pre- and post-processing and running Chicago in batch mode is also publicly available [45]. Chicago v1.0.1 was used in this paper.

A typical Chicago job for two biological replicates of $\mathrm{CHi}-\mathrm{C}$ data takes $2-3 \mathrm{~h}$ wall-clock time (including sample pre-processing from bam files using chicagoTools) and uses 50 GB RAM. An example workflow in the form of an $\mathrm{R}$ package vignette is provided as Additional file 3 . The description of free parameters and rationale for their settings is given in Additional file 2: Table S1.

\section{Assessment of feature enrichment}

Enrichment for chromatin features at $\mathrm{CHi}-\mathrm{C}$ interacting regions was assessed with respect to random HindIII fragments drawn in such a way as to match the distribution of the observed interaction distances. A $95 \%$ confidence interval for the expected overlap was obtained from 100 random draws. SNP enrichment at promoter interacting fragments was assessed using GoShifter [23].

\section{Hi-C analyses}

HOMER [28] was used to compute binned coverageand distance-related background in the $\mathrm{Hi}-\mathrm{C}$ data and call significantly interacting bin pairs. Short-range cis- chromosomal interactions $(<1 \mathrm{Mb})$ were detected in $25-\mathrm{kb}$ bins; long-range cis-chromosomal $(>1 \mathrm{Mb})$ and transchromosomal interactions were detected in $1-\mathrm{Mb}$ bins. Bin pairs with FDR-adjusted $p<0.05$ were considered significant. The significance of overlap between $\mathrm{CHi}-\mathrm{C}$ promoterinteracting regions identified by $\mathrm{CHiCAGO}$ and the HOMER-detected interacting bin pairs in the $\mathrm{Hi}-\mathrm{C}$ data was ascertained by permutation, while preserving the structural features of the data, as follows. Cis-chromosomal interactions were permuted across the baits while preserving the interaction distances. Trans-chromosomal interactions were permuted across chromosomes while preserving the relative chromosomal position of the interacting fragments.

\section{Data access}

Raw $\mathrm{CHi}-\mathrm{C}, \mathrm{Hi}-\mathrm{C}$ and random ligation control data used in this study are available in ArrayExpress $[54,55]$ under accession numbers E-MTAB-2323 (GM12878) and EMTAB-2414 (mESC), respectively. CHiCAGO experiment design files and output files produced with default package settings for GM12878 and mESCs are available through the Open Science Framework [56]. The interaction calls and raw reads for both cell types (score $\geq 5$ ) have also been submitted to the NCBI Gene Expression Omnibus under accession number GSE81503 [57].

\section{Additional files}

Additional file 1: The mathematical specification of the CHiCAGO algorithm. (PDF $304 \mathrm{~kb}$ )

Additional file 2: Figures S1 to S6 and Table S1. (PDF $803 \mathrm{~kb}$ )

Additional file 3: The CHiCAGO R package tutorial. (PDF 946 kb)

\section{Acknowledgements}

The authors would like to thank Simon Andrews, Chris Wallace, Oliver Burren and all members of the Spivakov, Fraser and Babraham Bioinformatics groups for helpful discussions. We are grateful to all our "wet-lab" collaborators (in particular, Mayra Furlan-Magaril, Mattia Frontini, Peter Rugg-Gunn and Willem Ouwehand) for using and testing CHiCAGO. This work has been funded by the Biotechnology and Biological Sciences Research Council and the Medical Research Council of the UK; DZ is funded by the European Molecular Biology Laboratory. Finally, we thank Laura Biggins for disambiguating the last two letters of CHiCAGO.

\section{Authors' contributions}

JC, PFP and MS designed the CHiCAGO algorithm; VP and DZ contributed statistical advice; JC, PFP, SWW and MS implemented the algorithm. SS, CO, $\mathrm{BMJ}$ and PF generated Capture Hi-C data and advised on their biological properties. PFP, CV, AD, JC and MS performed downstream validation analyses. JC, PFP and MS wrote the paper with critical input from all authors. MS supervised the work. All authors read and approved the final manuscript.

\section{Competing interests}

The authors declare that they have no competing interests.

Ethics approval and consent to participate

Ethics approval was not required for this study.

\section{Author details}

${ }^{1}$ Nuclear Dynamics Programme, Babraham Institute, Cambridge, UK.

${ }^{2}$ Bioinformatics Group, Babraham Institute, Cambridge, UK. ${ }^{3}$ UCL Genetics 
Institute, London, UK. ${ }^{4}$ European Molecular Biology Laboratory, European Bioinformatics Institute, Cambridge, UK. ${ }^{5}$ Department of Medical and Molecular Genetics, King's College, London, UK.

\section{Received: 1 April 2016 Accepted: 25 May 2016}

\section{Published online: 15 June 2016}

\section{References}

1. Dekker J, Marti-Renom MA, Mirny LA. Exploring the three-dimensional organization of genomes: interpreting chromatin interaction data. Nat Rev Genet. 2013;14:390-403.

2. van Steensel B, Dekker J. Genomics tools for unraveling chromosome architecture. Nat Biotechnol. 2010:28:1089-95.

3. Mifsud B, Tavares-Cadete F, Young AN, Sugar R, Schoenfelder S, Ferreira L, et al. Mapping long-range promoter contacts in human cells with high-resolution capture Hi-C. Nat Genet. 2015;47:598-606.

4. Schoenfelder S, Furlan-Magaril M, Mifsud B, Tavares-Cadete F, Sugar R, Javierre BM, et al. The pluripotent regulatory circuitry connecting promoters to their long-range interacting elements. Genome Res. 2015; 25:582-97.

5. Schoenfelder S, Sugar R, Dimond A, Javierre BM, Armstrong H, Mifsud B, et al. Polycomb repressive complex PRC1 spatially constrains the mouse embryonic stem cell genome. Nat Genet. 2015;47:1179-86.

6. Dryden NH, Broome LR, Dudbridge F, Johnson N, Orr N, Schoenfelder S, et al. Unbiased analysis of potential targets of breast cancer susceptibility loci by Capture Hi-C. Genome Res. 2014:24:1854-68.

7. Jager R, Migliorini G, Henrion M, Kandaswamy R, Speedy HE, Heindl A, et al. Capture $\mathrm{Hi}-\mathrm{C}$ identifies the chromatin interactome of colorectal cancer risk loci. Nat Commun. 2015:6:6178.

8. Imakaev M, Fudenberg G, McCord RP, Naumova N, Goloborodko A, Lajoie $\mathrm{BR}$, et al. Iterative correction of $\mathrm{Hi}-\mathrm{C}$ data reveals hallmarks of chromosome organization. Nat Methods. 2012;9:999-1003.

9. Sanyal A, Lajoie BR, Jain G, Dekker J. The long-range interaction landscape of gene promoters. Nature. 2012;489:109-13.

10. Yaffe $\mathrm{E}$, Tanay A. Probabilistic modeling of $\mathrm{Hi}-\mathrm{C}$ contact maps eliminates systematic biases to characterize global chromosomal architecture. Nat Genet. 2011:43:1059-65.

11. Sahlen P, Abdullayev I, Ramskold D, Matskova L, Rilakovic N, Lotstedt B, et al. Genome-wide mapping of promoter-anchored interactions with close to single-enhancer resolution. Genome Biol. 2015;16:156.

12. van de Werken HJ, Landan G, Holwerda SJ, Hoichman M, Klous P, Chachik R, et al. Robust 4C-seq data analysis to screen for regulatory DNA interactions. Nat Methods. 2012;9:969-72.

13. Klein FA, Pakozdi T, Anders S, Ghavi-Helm Y, Furlong EE, Huber W. FourCSeq: analysis of 4C sequencing data. Bioinformatics. 2015;31:3085-91.

14. Raviram R, Rocha PP, Müller CL, Miraldi ER, Badri S, Fu Y, et al. 4C-ker: a method to reproducibly identify genome-wide interactions captured by 4C-Seq experiments. PLoS Comput Biol. 2016;12:e1004780.

15. Genovese $C R$, Roeder $K$, Wasserman L. False discovery control with $p$-value weighting. Biometrika. 2006:93:509-24.

16. Ay F, Bailey TL, Noble WS. Statistical confidence estimation for Hi-C data reveals regulatory chromatin contacts. Genome Res. 2014;24:999-1011.

17. Thongjuea S, Stadhouders R, Grosveld FG, Soler E, Lenhard B. r3Cseq: an R/ Bioconductor package for the discovery of long-range genomic interactions from chromosome conformation capture and next-generation sequencing data. Nucleic Acids Res. 2013;41:e132.

18. Rosa A, Becker NB, Everaers R. Looping probabilities in model interphase chromosomes. Biophys J. 2010;98:2410-9.

19. Bohn M, Heermann DW. Diffusion-driven looping provides a consistent framework for chromatin organization. PLoS One. 2010;5:e12218.

20. Gui J, Tosteson T, Borsuk M. Weighted multiple testing procedures for genomic studies. BioData Mining. 2012;5:4

21. Ong C-T, Corces VG. CTCF: an architectural protein bridging genome topology and function. Nat Rev Genet. 2014;15:234-46.

22. Maurano MT, Humbert R, Rynes E, Thurman RE, Haugen E, Wang $H$, et al. Systematic localization of common disease-associated variation in regulatory DNA. Science. 2012;337:1190-5.

23. Trynka G, Westra H-J, Slowikowski K, Hu X, Xu H, Stranger Barbara E, et al. Disentangling the effects of colocalizing genomic annotations to functionally prioritize non-coding variants within complex-trait loci. Am J Hum Genet. 2015;97:139-52.
24. Akhtar W, de Jong J, Pindyurin AV, Pagie L, Meuleman W, de Ridder J, et al. Chromatin position effects assayed by thousands of reporters integrated in parallel. Cell. 2013;154:914-27.

25. Sexton $T$, Umlauf $D$, Kurukuti $S$, Fraser $P$. The role of transcription factories in large-scale structure and dynamics of interphase chromatin. Semin Cell Dev Biol. 2007;18:691-7.

26. Ma T, Van Tine BA, Wei Y, Garrett MD, Nelson D, Adams PD, et al. Cell cycleregulated phosphorylation of p220(NPAT) by cyclin E/Cdk2 in Cajal bodies promotes histone gene transcription. Genes Dev. 2000;14:2298-313.

27. Wang Q, Sawyer IA, Sung M-H, Sturgill D, Shevtsov SP, Pegoraro G, et al. Cajal bodies are linked to genome conformation. Nat Commun. 2016;7:10966.

28. Heinz S, Benner C, Spann N, Bertolino E, Lin YC, Laslo P, et al. Simple combinations of lineage-determining transcription factors prime cis-regulatory elements required for macrophage and B cell identities. Mol. Cell. 38:576-89.

29. Mifsud B, Martincorena I, Darbo E, Sugar R, Schoenfelder S, Fraser P, Luscombe N. GOTHiC, a simple probabilistic model to resolve complex biases and to identify real interactions in Hi-C data. biorXiv preprint. 2015:10.1101/023317.

30. Hu M, Deng K, Selvaraj S, Qin Z, Ren B, Liu JS. HiCNorm: removing biases in Hi-C data via Poisson regression. Bioinformatics. 2012;28:3131-3.

31. Dudoit S, van der Laan MJ. Multiple testing procedures with applications to genomics. New York: Springer; 2008.

32. Sun L, Craiu RV, Paterson AD, Bull SB. Stratified false discovery control for large-scale hypothesis testing with application to genome-wide association studies. Genet Epidemiol. 2006;30:519-30.

33. Lin WY, Lee WC. Improving power of genome-wide association studies with weighted false discovery rate control and prioritized subset analysis. PLoS One. 2012;7:e33716

34. Roeder K, Wasserman L. Genome-wide significance levels and weighted hypothesis testing. Stat Sci. 2009;24:398-413.

35. Li L, Kabesch $M$, Bouzigon $E$, Demenais F, Farrall M, Moffatt MF, et al. Using eQTL weights to improve power for genome-wide association studies: a genetic study of childhood asthma. Front Genet. 2013:4:103.

36. Ignatiadis N, Klaus B, Zaugg JB, Huber W. Data-driven hypothesis weighting increases detection power in genome-scale multiple testing. Nat. Methods. 2016; doi:10.1038/nmeth.3885.

37. Zhao H, Fung WK. A powerful FDR control procedure for multiple hypotheses. Comput Stat Data Anal. 2016;98:60-70.

38. Li Q, Brown JB, Huang H, Bickel PJ. Measuring reproducibility of high-throughput Ann Appl Stat. 2011;5:1752-79.

39. Benjamini $Y$, Hochberg $Y$. Controlling the false discovery rate: a practical and powerful approach to multiple testing. J R Stat Soc Ser B Methodol. 1995;57:289-300.

40. Love MI, Huber W, Anders S. Moderated estimation of fold change and dispersion for RNA-seq data with DESeq2. Genome Biol. 2014;15:1-21.

41. Jeffries CD, Ward WO, Perkins DO, Wright FA. Discovering collectively informative descriptors from high-throughput experiments. BMC Bioinformatics. 2009:10:431.

42. Blangiardo M, Cassese A, Richardson S. sdef: an R package to synthesize lists of significant features in related experiments. BMC Bioinformatics. 2010;11:1-10

43. Xu Z, Zhang G, Jin F, Chen M, Furey TS, Sullivan PF, et al. A hidden Markov random field-based Bayesian method for the detection of long-range chromosomal interactions in Hi-C data. Bioinformatics. 2016:32:650-6.

44. Dixon JR, Selvaraj S, Yue F, Kim A, Li Y, Shen Y, et al. Topological domains in mammalian genomes identified by analysis of chromatin interactions. Nature. 2012;485:376-80.

45. The CHiCAGO home page. http://www.regulatorygenomicsgroup.org/ chicago.Accessed 25 May 2016

46. HiCUP. http://www.bioinformatics.babraham.ac.uk/projects/hicup/ overview/. Accessed 25 May 2016.

47. Wingett $S$, Ewels $P$, Furlan-Magaril M, Nagano $T$, Schoenfelder $S$, Fraser $P$, et al. HiCUP: pipeline for mapping and processing Hi-C data. F1000Res. 2015:4:1310.

48. Anders S, Huber W. Differential expression analysis for sequence count data. Genome Biol. 2010;11:R106

49. Nelder JA, Mead R. A simplex method for function minimization. Comput J. 1965;7:308-13

50. R Development Core Team. R: a language and environment for statistical computing. Vienna, Austria: R Foundation for Statistical Computing; 2015.

51. data.table: Extension of data.frame. http://CRAN.R-project.org/package=data table. Accessed 25 May 2016. 
52. Huber W, Carey VJ, Gentleman R, Anders S, Carlson M, Carvalho BS, et al. Orchestrating high-throughput genomic analysis with Bioconductor. Nat Methods. 2015;12:115-21.

53. The Chicago R package on Bioconductor. http://bioconductor.org/ packages/release/bioc/html/Chicago.html. Accesseed 25 May 2016.

54. ArrayExpress-functional genomics data. https://www.ebi.ac.uk/ arrayexpress/. Accessed 25 May 2016.

55. Kolesnikov N, Hastings E, Keays M, Melnichuk O, Tang YA, Williams E, et al. ArrayExpress update-simplifying data submissions. Nucleic Acids Res. 2015; 43:D1113-6.

56. Analysis of Promoter Capture Hi-C data for GM12878 and mouse ES cells using the CHiCAGO pipeline. http://osf.io/nemc6. Accessed 18 May 2016.

57. NCBI Gene Expression Omnibus. http://www.ncbi.nlm.nih.gov/geo/query/ acc.cgi?acc=GSE81503. Accessed 25 May 2016.

58. WashU Epigenome Browser. http://epigenomegateway.wustl.edu. Accessed 25 May 2016.

59. Zhou X, Lowdon RF, Li D, Lawson HA, Madden PA, Costello JF, et al. Exploring long-range genome interactions using the WashU Epigenome Browser. Nat Methods. 2013;10:375-6.

60. Ernst J, Kellis M. ChromHMM: automating chromatin-state discovery and characterization. Nat Methods. 2012;9:215-6.

61. ENCODE Consortium. An integrated encyclopedia of DNA elements in the human genome. Nature. 2012;489:57-74.

62. Yue F, Cheng Y, Breschi A, Vierstra J, Wu W, Ryba T, et al. A comparative encyclopedia of DNA elements in the mouse genome. Nature. 2014;515: 355-64.

\section{Submit your next manuscript to BioMed Central} and we will help you at every step:

- We accept pre-submission inquiries

- Our selector tool helps you to find the most relevant journal

- We provide round the clock customer support

- Convenient online submission

- Thorough peer review

- Inclusion in PubMed and all major indexing services

- Maximum visibility for your research

Submit your manuscript at www.biomedcentral.com/submit 